\title{
Pacific
}

Journal of

Mathematics

\section{ON MAXIMAL LINDENSTRAUSS SPACES}

Petr Petráček AND JiŘí SPURnÝ 


\title{
ON MAXIMAL LINDENSTRAUSS SPACES
}

\author{
PETR PETRÁČEK AND JiŘí SPURnÝ
}

\begin{abstract}
We solve a problem of Lacey (1973) by showing that there exist a metrizable compact space $K$ and a closed space $\mathcal{H} \subset \mathcal{C}(K)$ containing constants with $\overline{\partial_{\mathcal{H}} K}=K$ such that $\mathcal{H}$ is maximal with respect to $\partial_{\mathcal{H}} K$ and $\mathcal{H}$ is not a Lindenstrauss space.
\end{abstract}

\section{Introduction}

Let $X$ be a compact convex subset of a real locally convex space and let $\mathfrak{A}^{c}(X)$ denote the space of all affine continuous functions on $X$. Denote by ext $X$ the set of all extreme points of $X$.

Let $K$ be a compact Hausdorff topological space and $\mathcal{H} \subset \mathcal{C}(K)$ a closed subspace of $\mathcal{C}(K)$ containing constants and separating points of $K$. The space $\mathcal{H}$ can be identified with $\mathfrak{A}^{c}(X)$, where

$$
X=\left\{s^{*} \in \mathcal{H}^{*}: s^{*}(1)=\left\|s^{*}\right\|=1\right\}
$$

with the weak* topology. Consider the set

$$
\partial_{\mathcal{H}} K=\left\{x \in K:\left.\varepsilon_{x}\right|_{\mathcal{H}} \text { is an extreme point of the unit ball of } \mathcal{H}^{*}\right\},
$$

where $\varepsilon_{x}$ denotes the Dirac measure at $x \in K$. Then ext $X$ is homeomorphic to $\partial_{\mathcal{H}} K$ via the evaluation mapping (see Theorem 2.1 and [LMNS 2010, Proposition 4.26]).

The space $\mathcal{H}$ is called maximal with respect to $\partial_{\mathcal{H}} K$ if for every closed space $\mathcal{G}$ with $\mathcal{H} \subset \mathcal{G} \subset \mathcal{C}(K)$ we have $\mathcal{H}=\mathcal{G}$ provided $\partial_{\mathcal{H}} K=\partial_{\mathcal{G}} K$.

(In [Lacey 1973], the property of separating points is not a part of the definition of a function space. Nevertheless, in our opinion, this property is necessary for $\partial_{\mathcal{H}} K$ to be homeomorphic to ext $X$. Indeed, consider $\mathcal{H}=\operatorname{span}\{1\}$ on $[0,1]$. Then $X$ is a singleton, and thus $\partial_{\mathcal{H}}[0,1]=[0,1]$. Obviously, $[0,1]$ is not homeomorphic to ext $X$.)

It is shown in [Edwards and Vincent-Smith 1968] that $\mathcal{H}$ is maximal with respect to $\partial_{\mathcal{H}} K$ whenever $\mathcal{H}$ is a Lindenstrauss space; see Theorems 2.1 and 2.3 below.

The research was supported by grant GAČR P201/12/0290. Spurný was also supported by the Neuron Fund for Support of Science.

MSC2010: 46B25.

Keywords: Lindenstrauss space, $L_{1}$-predual, function space. 
(A real Banach space $X$ is called a Lindenstrauss space, or an $L_{1}$-predual, if its dual space $X^{*}$ is isometric to a space $L_{1}(X, \mathcal{S}, \mu)$ for some measure space $(X, \mathcal{S}, \mu)$.) This result serves as a motivation for the following problem, stated as Question 5 in [Lacey 1973, p. 144] (see also [Lacey 1974, p. 198]).

Question 1.1. Let $K$ be a compact space and $\mathcal{H} \subset \mathcal{C}(K)$ a closed subspace containing constants and separating points of $K$ such that $\overline{\partial_{\mathcal{H}} K}=K$. Let $\mathcal{H}$ be maximal with respect to $\partial_{\mathcal{H}} K$. Is $\mathcal{H}$ then a Lindenstrauss space?

The aim of our paper is to show that the answer to Question 1.1 is in general negative by proving the following theorem.

Theorem 1.2. There exist a metrizable compact space $K$ and a closed space $\mathcal{H} \subset \mathcal{C}(K)$ containing constants and separating points of $K$ with $\overline{\partial_{\mathcal{H}} K}=K$ such that $\mathcal{H}$ is maximal with respect to $\partial_{\mathcal{H}} K$ and $\mathcal{H}$ is not a Lindenstrauss space.

\section{Function spaces}

Let $K$ be a compact space (we consider all topological spaces as Hausdorff). We identify the dual of $\mathcal{C}(K)$ with the space $\mathcal{M}(K)$ of all signed Radon measures on $K$. By a positive Radon measure on $K$ we mean a finite complete inner regular measure defined at least on all Borel subsets of $K$. Let $\mathcal{M}^{1}(K)$ denote the set of all probability Radon measures on $K, \mathcal{M}^{+}(K)$ the set of all positive Radon measures on $K$, and $\varepsilon_{x}$ the Dirac measure at $x \in K$.

By a function space $\mathcal{H}$ on $K$ we mean a subspace $\mathcal{H}$ of $\mathcal{C}(K)$ containing constants and separating points of $K$. Assuming $\mathcal{H}$ is a function space on $K$ we assign to each $x \in K$ the set

$$
\mathcal{M}_{x}(\mathcal{H})=\left\{\mu \in \mathcal{M}^{1}(K): \mu(h)=h(x), h \in \mathcal{H}\right\}
$$

of all $\mathcal{H}$-representing measures. Clearly, $\varepsilon_{x} \in \mathcal{M}_{x}(\mathcal{H})$ for each $x \in K$. We call

$$
\mathrm{Ch}_{\mathcal{H}} K=\left\{x \in K: \mathcal{M}_{x}(\mathcal{H})=\left\{\varepsilon_{x}\right\}\right\}
$$

the Choquet boundary of $\mathcal{H}$. If $h \in \mathcal{H}$ attains its strict minimum at some $x \in K$, we call $h$ an $\mathcal{H}$-exposing function and $x$ an $\mathcal{H}$-exposed point. It is easy to see that any $\mathcal{H}$-exposed point belongs to the Choquet boundary of $\mathcal{H}$.

We define the space $\mathcal{A}^{c}(\mathcal{H})$ of all continuous $\mathcal{H}$-affine functions to be the family of all continuous functions $f$ on $K$ satisfying

$$
f(x)=\int_{K} f d \mu \quad \text { for each } x \in K \text { and } \mu \in \mathcal{M}_{x}(\mathcal{H}) .
$$

$\mathcal{A}^{c}(\mathcal{H})$ is a closed function space containing $\mathcal{H}$ and satisfying $\mathcal{M}_{x}(\mathcal{H})=\mathcal{M}_{x}\left(\mathcal{A}^{c}(\mathcal{H})\right)$ for every $x \in K$. Thus $\mathrm{Ch}_{\mathcal{H}} K=\mathrm{Ch}_{\mathcal{A}^{c}(\mathcal{H})} K$. We define the state space of $\mathcal{H}$ as

$$
\boldsymbol{S}(\mathcal{H})=\left\{s^{*} \in \mathcal{H}^{*}: s(1)=\|s\|=1\right\}
$$


endowed with the weak* topology. The state space $S(\mathcal{H})$ is a compact convex set and $K$ is homeomorphically embedded into $S(\mathcal{H})$ via $\phi: K \rightarrow S(\mathcal{H})$, where

$$
\phi(x): h \rightarrow h(x), \quad h \in \mathcal{H}, x \in K .
$$

Let $B_{\mathcal{H}^{*}}$ stand for the unit ball of $\mathcal{H}^{*}$. Following the notation in [Lacey 1973, p. 143] mentioned in the introduction,

$$
\partial_{\mathcal{H}} K=\left\{x \in K: \phi(x) \in \operatorname{ext} B_{\mathcal{H}^{*}}\right\} .
$$

The next assertion shows that our definition of the Choquet boundary coincides with Lacey's definition of $\partial_{\mathcal{H}} K$.

Theorem 2.1. If $\mathcal{H}$ is a function space on a compact space $K$, then $\mathrm{Ch}_{\mathcal{H}} K=\partial_{\mathcal{H}} K$. Proof. By [LMNS 2010, Proposition 4.26(d)], $\phi\left(\mathrm{Ch}_{\mathcal{H}} K\right)=\operatorname{ext} \boldsymbol{S}(\mathcal{H})$. Since $\boldsymbol{S}(\mathcal{H})$ is a face of $B_{\mathcal{H}^{*}}$ (see [LMNS 2010, Section 2.3.A]), we have ext $\boldsymbol{S}(\mathcal{H})=$ ext $B_{\mathcal{H}^{*}} \cap \boldsymbol{S}(\mathcal{H})$. Thus, given any $x \in K$, we have $\phi(x) \in \operatorname{ext} \boldsymbol{S}(\mathcal{H})$ if and only if $\phi(x) \in \operatorname{ext} B_{\mathcal{H}^{*}}$.

The Choquet ordering on $\mathcal{M}^{+}(K)$ is given as follows: $\mu \prec v$ if $\mu(k) \leq v(k)$ for each function $k$ of the form $k=\max \left\{h_{1}, \ldots, h_{n}\right\}$, where $n \in \mathbb{N}$ and $h_{1}, \ldots, h_{n} \in \mathcal{H}$ (see [LMNS 2010, Definition 3.19 and Proposition 3.56]). A measure $\mu$ in $\mathcal{M}^{+}(K)$ is called $\mathcal{H}$-maximal if it is $\prec-$ maximal. By [LMNS 2010, Theorem 3.65], there exists an $\mathcal{H}$-maximal measure $\mu \in \mathcal{M}_{x}(\mathcal{H})$ for every $x \in K$. Furthermore, if $K$ is metrizable, the set $\mathrm{Ch}_{\mathcal{H}} K$ is $G_{\delta}$ (see [LMNS 2010, Theorem 3.42 and Proposition 3.43]) and $\mathcal{H}$-maximal measures are precisely those measures carried by $\mathrm{Ch}_{\mathcal{H}} K$ (see [LMNS 2010, Corollary 3.62]).

If for each $x \in K$ there exists only one $\mathcal{H}$-maximal measure in $\mathcal{M}_{x}(\mathcal{H})$, the function space $\mathcal{H}$ is called simplicial (see [LMNS 2010, Chapter 6]). A compact convex set $X$ is called a simplex if the function space $\mathfrak{A}^{c}(X)$ is simplicial. The relation between simplicial function spaces and Lindenstrauss spaces is given by the following result.

Theorem 2.2. Let $\mathcal{H}$ be a function space on a compact space $K$. Then $\mathcal{H}$ is simplicial if and only if the Banach space $\mathcal{A}^{c}(\mathcal{H})$ is a Lindenstrauss space.

Proof. Let $\mathcal{A}^{c}(\mathcal{H})$ be a Lindenstrauss space. Since $\mathfrak{A}^{c}\left(S\left(\mathcal{A}^{c}(\mathcal{H})\right)\right)$ is isometric to the space $\mathcal{A}^{c}(\mathcal{H})$ (see [LMNS 2010, Proposition 4.26]), it is a Lindenstrauss space as well. By [Fonf et al. 2001, Proposition 3.23], $\boldsymbol{S}\left(\mathcal{A}^{c}(\mathcal{H})\right)$ is a simplex. Thus it follows from [LMNS 2010, Theorem 6.54] that $\mathcal{H}$ is simplicial.

Conversely, if $\mathcal{H}$ is simplicial, $\boldsymbol{S}\left(\mathcal{A}^{c}(\mathcal{H})\right)$ is a simplex by [LMNS 2010, Theorem 6.54]. Using [Fonf et al. 2001, Proposition 3.23] we conclude that $\mathcal{A}^{c}(\mathcal{H})$, being isometric to $\mathfrak{A}^{c}\left(S\left(\mathcal{A}^{c}(\mathcal{H})\right)\right)$, is a Lindenstrauss space. 
The next result asserts two important properties of closed function spaces that are Lindenstrauss spaces. As mentioned above, it can be considered a motivation for the question this paper aims to answer.

Theorem 2.3. Let $\mathcal{H}$ be a closed function space on a compact space $K$ such that $\mathcal{H}$ is a Lindenstrauss space. Then $\mathcal{H}=\mathcal{A}^{c}(\mathcal{H})$ and $\mathcal{H}$ is maximal with respect to $\mathrm{Ch}_{\mathcal{H}} K$.

Proof. To prove the first assertion notice that $\mathfrak{A}^{c}(\boldsymbol{S}(\mathcal{H})$ ), being isometric to $\mathcal{H}$ (see [LMNS 2010, Proposition 4.26]), is a Lindenstrauss space. By [Fonf et al. 2001, Proposition 3.23], $\boldsymbol{S}(\mathcal{H})$ is a simplex. This implies that $\mathfrak{A}^{c}(\boldsymbol{S}(\mathcal{H}))$ is simplicial and thus, by [LMNS 2010, Theorem 6.16(vi)], $\mathfrak{A}^{c}(\boldsymbol{S}(\mathcal{H}))$ has the so-called weak Riesz interpolation property. This, however, implies that $\mathcal{H}$ has the weak Riesz interpolation property according to [LMNS 2010, Proposition 4.26]. To finish the proof it is enough to consult [LMNS 2010, Exercise 6.78].

To prove the second assertion, let $\mathcal{G} \supset \mathcal{H}$ be a closed function space with $\mathrm{Ch}_{\mathcal{H}} K=\mathrm{Ch}_{\mathcal{G}} K$. Since $\mathcal{G} \subset \mathcal{A}^{c}(\mathcal{G})$ and $\mathrm{Ch}_{\mathcal{G}} K=\mathrm{Ch}_{\mathcal{A}^{c}(\mathcal{G})} K$, we can assume without loss of generality that $\mathcal{A}^{c}(\mathcal{G})=\mathcal{G}$. Using [LMNS 2010, Theorem 10.60] we infer that $\mathcal{G}=\mathcal{A}^{c}(\mathcal{H})$. Since $\mathcal{H}=\mathcal{A}^{c}(\mathcal{H})$, we get $\mathcal{G}=\mathcal{H}$, finishing the proof.

\section{Proof of Theorem 1.2}

We consider a compact subset of $\mathbb{R}^{2}$ defined as follows. Let $\left\{s, s^{1}, s^{2}, t^{1}, t^{2}\right\}$ be distinct points in $\mathbb{R}^{2}$. Let $\left(s_{n}^{i}\right)$ and $\left(t_{n}^{i}\right), i=0,1,2$, be sequences of points in $\mathbb{R}^{2}$ such that

- $s_{n}^{0} \rightarrow s, t_{n}^{0} \rightarrow s$,

- $s_{n}^{i} \rightarrow s^{i}, t_{n}^{i} \rightarrow t^{i}, i=1,2$,

- all the elements of these sequences are pairwise distinct and not contained in $\left\{s, s^{1}, s^{2}, t^{1}, t^{2}\right\}$.

Let $B(x, r)$ denote the closed ball in $\mathbb{R}^{2}$ with center $x \in \mathbb{R}^{2}$ and diameter $r>0$. Let further $r_{n}>0, n \in \mathbb{N}$, be numbers such that

- $r_{n} \rightarrow 0$,

- the family

$$
\mathcal{K}=\left\{\{s\},\left\{s^{1}\right\},\left\{s^{2}\right\},\left\{t^{1}\right\},\left\{t^{2}\right\}\right\} \cup\left\{B\left(s_{n}^{0}, r_{n}\right): n \in \mathbb{N}\right\} \cup\left\{B\left(t_{n}^{0}, r_{n}\right): n \in \mathbb{N}\right\}
$$

is disjoint. 
We define the compact space $K$ as $K=\bigcup \mathcal{K}$. Furthermore, we set $\mathcal{H}$ to be

$$
\begin{aligned}
\mathcal{H}=\left\{h \in \mathcal{C}(K): h(s)=\frac{1}{2}\left(h\left(s^{1}\right)+h\left(s^{2}\right)\right)=\frac{1}{2}\left(h\left(t^{1}\right)+h\left(t^{2}\right)\right),\right. \\
\left.h\left(s_{n}^{0}\right)=\frac{1}{2}\left(h\left(s_{n}^{1}\right)+h\left(s_{n}^{2}\right)\right), h\left(t_{n}^{0}\right)=\frac{1}{2}\left(h\left(t_{n}^{1}\right)+h\left(t_{n}^{2}\right)\right), n \in \mathbb{N}\right\} .
\end{aligned}
$$

Lemma 3.1. The space $\mathcal{H}$ is a well defined function space with $\mathcal{H}=\mathcal{A}^{c}(\mathcal{H})$. Let $L=\{s\} \cup\left\{s_{n}^{0}: n \in \mathbb{N}\right\} \cup\left\{t_{n}^{0}: n \in \mathbb{N}\right\}$. Then $\mathrm{Ch}_{\mathcal{H}} K=K \backslash L$. In particular, $\mathrm{Ch}_{\mathcal{H}} K$ is dense in $K$.

Proof. Obviously, $\mathcal{H}$ contains constant functions. The fact that $\mathcal{H}=\mathcal{A}^{c}(\mathcal{H})$ follows immediately from the definition of $\mathcal{H}$. To verify that $\mathcal{H}$ separates points of $K$ it is enough to consider elementary constructions of functions from $\mathcal{H}$. Given $n \in \mathbb{N}$ and $z \in B\left(s_{n}^{0}, r_{n}\right) \backslash\left\{s_{n}^{0}\right\}$, we consider a continuous function $g: B\left(s_{n}^{0}, r_{n}\right) \rightarrow[0,1]$ attaining 0 precisely at $z$ and 1 at $s_{n}^{0}$. Then the function

$$
h_{z}(x)= \begin{cases}g(x) & \text { if } x \in B\left(s_{n}^{0}, r_{n}\right), \\ 1 & \text { otherwise }\end{cases}
$$

separates $z$ from the remaining points of $K$. It also $\mathcal{H}$-exposes $z$, and thus $z \in \mathrm{Ch}_{\mathcal{H}} K$.

We can further construct functions $h_{s_{n}}$ and $h_{s}$ in $\mathcal{H}$ as follows:

$$
\begin{aligned}
& h_{s_{n}}(x)= \begin{cases}0 & \text { if } x=s_{n}^{1}, \\
2 & \text { if } x \in B\left(s_{n}^{0}, r_{n}\right), \\
4 & \text { if } x=s_{n}^{2}, \\
1 & \text { otherwise, }\end{cases} \\
& h_{s}(x)= \begin{cases}0 & \text { if } x=s^{1}, \\
2 & \text { if } x=s^{2}, \\
\frac{1}{2 n} & \text { if } x=s_{n}^{1}, \\
2-\frac{1}{2 n} & \text { if } x=s_{n}^{2}, \\
1 & \text { otherwise. }\end{cases}
\end{aligned}
$$

The function $h_{s_{n}}$ then separates the points $s_{n}^{1}, s_{n}^{2}$ from any point in $K$ and it separates $s_{n}^{0}$ from any point in $K \backslash B\left(s_{n}^{0}, r_{n}\right)$. Its construction also shows that the points $s_{n}^{1}, s_{n}^{2}$ are $\mathcal{H}$-exposed and thus lie in $\mathrm{Ch}_{\mathcal{H}} K$. Similarly, the function $h_{s}$ separates points $s^{1}, s, s^{2}$ from each other and it separates $s$ from every point in $\left\{s_{n}^{i}: n \in \mathbb{N}, i \in\{1,2\}\right\}$. Furthermore, the construction of $h_{s}$ shows that the points $s^{1}, s^{2}$ are $\mathcal{H}$-exposed and thus belong to $\mathrm{Ch}_{\mathcal{H}} K$.

Analogously we can construct functions $h_{t_{n}}, \tilde{h}_{s}$ and $h_{y}$ for any $n \in \mathbb{N}$ and $y \in$ $B\left(t_{n}^{0}, r_{n}\right) \backslash\left\{t_{n}^{0}\right\}$ to show that $\mathcal{H}$ indeed separates points of $K$ and that all points in

$$
\left\{t_{1}, t_{2}\right\} \cup\left\{t_{n}^{i}: n \in \mathbb{N}, i \in\{1,2\}\right\} \cup \bigcup_{n \in \mathbb{N}}\left(B\left(t_{n}^{0}, r_{n}\right) \backslash\left\{t_{n}^{0}\right\}\right)
$$

lie in $\mathrm{Ch}_{\mathcal{H}} K$. 
Overall, we have

$$
\left\{s^{1}, s^{2}, t^{1}, t^{2}\right\} \cup\left\{s_{n}^{i}: n \in \mathbb{N}, i \in\{1,2\}\right\} \cup\left\{t_{n}^{i}: n \in \mathbb{N}, i \in\{1,2\}\right\} \subset \mathrm{Ch}_{\mathcal{H}} K
$$

and

$$
\bigcup_{n \in \mathbb{N}}\left(B\left(s_{n}^{0}, r_{n}\right) \backslash\left\{s_{n}^{0}\right\}\right) \cup \bigcup_{n \in \mathbb{N}}\left(B\left(t_{n}^{0}, r_{n}\right) \backslash\left\{t_{n}^{0}\right\}\right) \subset \mathrm{Ch}_{\mathcal{H}} K .
$$

Clearly, any point in $L$ has a nontrivial $\mathcal{H}$-representing measure. This together with the inclusions above yields $\mathrm{Ch}_{\mathcal{H}} K=K \backslash L$.

Lemma 3.2. Let $n \in \mathbb{N}$. Then

$$
\mathcal{M}_{s_{n}^{0}}(\mathcal{H})=\operatorname{conv}\left\{\varepsilon_{s_{n}^{0}}, \frac{1}{2}\left(\varepsilon_{s_{n}^{1}}+\varepsilon_{s_{n}^{2}}\right)\right\}
$$

and

$$
\mathcal{M}_{t_{n}^{0}}(\mathcal{H})=\operatorname{conv}\left\{\varepsilon_{t_{n}^{0}}, \frac{1}{2}\left(\varepsilon_{t_{n}^{1}}+\varepsilon_{t_{n}^{2}}\right)\right\} .
$$

Proof. Let $n \in \mathbb{N}$ and $\mu \in \mathcal{M}_{s_{n}^{0}}(\mathcal{H})$ be fixed. Pick a continuous function

$$
g: B\left(s_{n}^{0}, r_{n}\right) \rightarrow[0,1]
$$

such that $g\left(s_{n}^{0}\right)=0$ and $g(x)>0$ otherwise. Using the function

$$
h(x)= \begin{cases}0 & \text { if } x \in\left\{s_{n}^{1}, s_{n}^{2}\right\}, \\ g(x) & \text { if } x \in B\left(s_{n}^{0}, r_{n}\right), \\ 1 & \text { otherwise, }\end{cases}
$$

we infer that the support of $\mu$ is contained in $\left\{s_{n}^{0}, s_{n}^{1}, s_{n}^{2}\right\}$.

Further, let $a=\mu\left(\left\{s_{n}^{0}\right\}\right)$. Assume first that $a=0$, i.e., $\mu=b \varepsilon_{s_{n}^{1}}+(1-b) \varepsilon_{s_{n}^{2}}$ for some $b \in[0,1]$. Then the function

$$
h(x)= \begin{cases}0 & \text { if } x=s_{n}^{1} \\ 1 & \text { if } x=s_{n}^{0} \\ 2 & \text { if } x=s_{n}^{2} \\ 1 & \text { otherwise }\end{cases}
$$

shows that

$$
1=h\left(s_{n}^{0}\right)=\mu(h)=(1-b) h\left(s_{n}^{2}\right)=(1-b) 2 .
$$

In other words, $b=\frac{1}{2}$ and $\mu=\frac{1}{2}\left(\varepsilon_{s_{n}^{1}}+\varepsilon_{s_{n}^{2}}\right)$.

If $a \in(0,1)$, then the measure $v=\mu-a \varepsilon_{s_{n}^{0}}$ satisfies

$$
h\left(s_{n}^{0}\right)=\mu(h)=v(h)+a h\left(s_{n}^{0}\right), \quad h \in \mathcal{H} .
$$

Hence $\frac{1}{1-a} v$ is in $\mathcal{M}_{s_{n}^{0}}(\mathcal{H})$ and is carried by $\left\{s_{n}^{1}, s_{n}^{2}\right\}$. By the first part of the proof,

$$
\frac{1}{1-a} v=\frac{1}{2}\left(\varepsilon_{s_{n}^{1}}+\varepsilon_{s_{n}^{2}}\right) \text {. }
$$


Thus

$$
\mu=v+a \varepsilon_{s_{n}^{0}}=(1-a) \frac{1}{1-a} v+a \varepsilon_{s_{n}^{0}}=(1-a) \frac{1}{2}\left(\varepsilon_{s_{n}^{1}}+\varepsilon_{s_{n}^{2}}\right)+a \varepsilon_{s_{n}^{0}}
$$

is in $\operatorname{conv}\left\{\varepsilon_{s_{n}^{0}}, \frac{1}{2}\left(\varepsilon_{s_{n}^{1}}+\varepsilon_{s_{n}^{2}}\right)\right\}$. If $a=1$, obviously

$$
\mu=\varepsilon_{s_{n}^{0}} \in \operatorname{conv}\left\{\varepsilon_{s_{n}^{0}}, \frac{1}{2}\left(\varepsilon_{s_{n}^{1}}+\varepsilon_{s_{n}^{2}}\right)\right\} .
$$

Thus

$$
\mu \in \operatorname{conv}\left\{\varepsilon_{s_{n}^{0}}, \frac{1}{2}\left(\varepsilon_{s_{n}^{1}}+\varepsilon_{s_{n}^{2}}\right)\right\}
$$

holds in all cases.

The second part of the assertion can be proved analogously.

Lemma 3.3. The space $\mathcal{H}$ is not simplicial.

Proof. The measures $\frac{1}{2}\left(\varepsilon_{s^{1}}+\varepsilon_{s^{2}}\right), \frac{1}{2}\left(\varepsilon_{t^{1}}+\varepsilon_{t^{2}}\right)$ are different, they $\mathcal{H}$-represent $s$ and, by Lemma 3.1, both are carried by $\mathrm{Ch}_{\mathcal{H}} K$. Hence there exist two $\mathcal{H}$-maximal measures representing $s$, which implies that $\mathcal{H}$ is not simplicial.

Lemma 3.4. The space $\mathcal{H}$ is maximal with respect to $\mathrm{Ch}_{\mathcal{H}} K$. That is, $\mathcal{G}=\mathcal{H}$ for any closed function space $\mathcal{H} \subset \mathcal{G}$ such that $\mathrm{Ch}_{\mathcal{G}} K=\mathrm{Ch}_{\mathcal{H}} K$.

Proof. Fix an index $m \in \mathbb{N}$. Let $\tau \in \mathcal{M}_{s_{m}^{0}}(\mathcal{G})$ be a measure carried by $\mathrm{Ch}_{\mathcal{G}} K$. We aim to show that

$$
\tau=\frac{1}{2}\left(\varepsilon_{s_{m}^{1}}+\varepsilon_{s_{m}^{2}}\right) .
$$

Since $\mathcal{M}_{s_{m}^{0}}(\mathcal{G}) \subset \mathcal{M}_{s_{m}^{0}}(\mathcal{H})$, we obtain by virtue of Lemma 3.2 that

$$
\tau \in \operatorname{conv}\left\{\varepsilon_{S_{m}^{0}}, \frac{1}{2}\left(\varepsilon_{s_{m}^{1}}+\varepsilon_{S_{m}^{2}}^{2}\right)\right\} .
$$

This and the fact that $\tau$ is carried by $\mathrm{Ch}_{\mathcal{G}} K=\mathrm{Ch}_{\mathcal{H}} K \subset K \backslash\left\{s_{m}^{0}\right\}$ imply (3-1).

Pick $\mu_{n} \in \mathcal{M}_{s_{n}^{0}}(\mathcal{G}), n \in \mathbb{N}$, such that the measures $\mu_{n}$ are carried by $\mathrm{Ch}_{\mathcal{G}} K$ for all $n \in \mathbb{N}$. The sequence $\left(s_{n}^{0}\right)$ converges to $s$, while the sequence $\left(\mu_{n}\right)$ converges to $\mu=\frac{1}{2}\left(\varepsilon_{s^{1}}+\varepsilon_{s^{2}}\right)$. Thus $\mu \in \mathcal{M}_{s}(\mathcal{G})$. Analogously we infer that any measure $v_{n}$ in $\mathcal{M}_{t_{n}^{0}}(\mathcal{G})$ carried by $\mathrm{Ch}_{\mathcal{G}} K$ satisfies $v_{n}=\frac{1}{2}\left(\varepsilon_{t_{n}^{1}}+\varepsilon_{t_{n}^{2}}\right)$, and thus $v=\frac{1}{2}\left(\varepsilon_{t^{1}}+\varepsilon_{t^{2}}\right)$ is in $\mathcal{M}_{s}(\mathcal{G})$.

We want to show that $\mathcal{G} \subset \mathcal{H}$. To this end, let $g \in \mathcal{G}$ be given. We have to verify the conditions defining the space $\mathcal{H}$. Using the arguments above we get

$$
g\left(s_{n}^{0}\right)=\mu_{n}(g)=\frac{1}{2}\left(g\left(s_{n}^{1}\right)+g\left(s_{n}^{2}\right)\right) \quad \text { and } \quad g(s)=\mu(g)=\frac{1}{2}\left(g\left(s^{1}\right)+g\left(s^{2}\right)\right),
$$

while simultaneously

$$
g\left(t_{n}^{0}\right)=v_{n}(g)=\frac{1}{2}\left(g\left(t_{n}^{1}\right)+g\left(t_{n}^{2}\right)\right) \quad \text { and } \quad g(s)=v(g)=\frac{1}{2}\left(g\left(t^{1}\right)+g\left(t^{2}\right)\right) .
$$

Hence $g \in \mathcal{H}$ by definition. This concludes the proof. 
Thus we have proved Theorem 1.2. Indeed, considering the compact space $K$ and the closed function space $\mathcal{H} \subset \mathcal{C}(K)$ defined above, we have by Lemma 3.1 that $\mathrm{Ch}_{\mathcal{H}} K$ is dense in $K$. Furthermore, $\mathcal{H}$ is maximal with respect to $\mathrm{Ch}_{\mathcal{H}} K$ by Lemma 3.4. Since $\mathcal{H}$ is not simplicial according to Lemma 3.3, Theorem 2.2 asserts that $\mathcal{A}^{c}(\mathcal{H})$ is not a Lindenstrauss space. Since $\mathcal{H}=\mathcal{A}^{c}(\mathcal{H})$ by Lemma 3.1, it follows that $\mathcal{H}$ is not a Lindenstrauss space.

\section{References}

[Edwards and Vincent-Smith 1968] D. A. Edwards and G. Vincent-Smith, "A Weierstrass-Stone theorem for Choquet simplexes", Ann. Inst. Fourier (Grenoble) 18:1 (1968), 261-282. MR 39 \#6060 Zbl 0172.15604

[Fonf et al. 2001] V. P. Fonf, J. Lindenstrauss, and R. R. Phelps, "Infinite dimensional convexity", pp. 599-670 in Handbook of the geometry of Banach spaces, vol. I, edited by W. B. Johnson and J. Lindenstrauss, North-Holland, Amsterdam, 2001. MR 2003c:46014 Zbl 1086.46004

[Lacey 1973] H. E. Lacey, "On the classification of Lindenstrauss spaces”, Pacific J. Math. 47 (1973), 139-145. MR 50 \#5443 Zbl 0251.46030

[Lacey 1974] H. E. Lacey, The isometric theory of classical Banach spaces, Die Grundlehren der mathematischen Wissenschaften 208, Springer, New York, 1974. MR 58 \#12308 Zbl 0285.46024

[LMNS 2010] J. Lukeš, J. Malý, I. Netuka, and J. Spurný, Integral representation theory: applications to convexity, Banach spaces and potential theory, de Gruyter Studies in Mathematics 35, de Gruyter, Berlin, 2010. MR 2011e:46002 Zbl 1216.46003

Received May 5, 2014.

PETR PETRÁČEK

DEPARTMENT OF MATHEMATICAL ANALYSis

FACULTY OF MATHEMATICS AND PHYSICS

CHARLES UNIVERSITY

SOKOLOVSKÁ 83

18675 PRAHA 8

CZECh RePublic

petracek@karlin.mff.cuni.cz

JIŘÍ SPURNÝ

DEPARTMENT OF MATHEMATICAL ANALYSIS

FACUlTy OF MATHEMATICS AND PHYSICS

CHARLES UNIVERSITY

SOKOLOVSKÁ 83

18675 PRAHA 8

CZECH REPUbLiC

spurny@karlin.mff.cuni.cz 


\title{
PACIFIC JOURNAL OF MATHEMATICS
}

\author{
msp.org/pjm
}

Founded in 1951 by E. F. Beckenbach (1906-1982) and F. Wolf (1904-1989)

\section{EDITORS}

Don Blasius (Managing Editor)

Department of Mathematics

University of California

Los Angeles, CA 90095-1555

blasius@math.ucla.edu

\author{
Paul Balmer \\ Department of Mathematics \\ University of California \\ Los Angeles, CA 90095-1555 \\ balmer@math.ucla.edu \\ Robert Finn \\ Department of Mathematics \\ Stanford University \\ Stanford, CA 94305-2125 \\ finn@math.stanford.edu \\ Sorin Popa \\ Department of Mathematics \\ University of California \\ Los Angeles, CA 90095-1555 \\ popa@math.ucla.edu
}

\author{
Vyjayanthi Chari \\ Department of Mathematics \\ University of California \\ Riverside, CA 92521-0135 \\ chari@math.ucr.edu \\ Kefeng Liu \\ Department of Mathematics \\ University of California \\ Los Angeles, CA 90095-1555 \\ liu@math.ucla.edu \\ Jie Qing \\ Department of Mathematics \\ University of California \\ Santa Cruz, CA 95064 \\ qing@ cats.ucsc.edu
}

\section{PRODUCTION}

Silvio Levy, Scientific Editor, production@msp.org

\section{SUPPORTING INSTITUTIONS}

ACADEMIA SINICA, TAIPEI

CALIFORNIA INST. OF TECHNOLOGY

INST. DE MATEMÁTICA PURA E APLICADA

KEIO UNIVERSITY

MATH. SCIENCES RESEARCH INSTITUTE

NEW MEXICO STATE UNIV.

OREGON STATE UNIV.

\author{
STANFORD UNIVERSITY \\ UNIV. OF BRITISH COLUMBIA \\ UNIV. OF CALIFORNIA, BERKELEY \\ UNIV. OF CALIFORNIA, DAVIS \\ UNIV. OF CALIFORNIA, LOS ANGELES \\ UNIV. OF CALIFORNIA, RIVERSIDE \\ UNIV. OF CALIFORNIA, SAN DIEGO \\ UNIV. OF CALIF., SANTA BARBARA
}

\author{
Daryl Cooper \\ Department of Mathematics \\ University of California \\ Santa Barbara, CA 93106-3080 \\ cooper@math.ucsb.edu \\ Jiang-Hua Lu \\ Department of Mathematics \\ The University of Hong Kong \\ Pokfulam Rd., Hong Kong \\ jhlu@maths.hku.hk \\ Paul Yang \\ Department of Mathematics \\ Princeton University \\ Princeton NJ 08544-1000 \\ yang@math.princeton.edu
}

These supporting institutions contribute to the cost of publication of this Journal, but they are not owners or publishers and have no responsibility for its contents or policies.

See inside back cover or msp.org/pjm for submission instructions.

The subscription price for 2015 is US \$420/year for the electronic version, and \$570/year for print and electronic.

Subscriptions, requests for back issues and changes of subscribers address should be sent to Pacific Journal of Mathematics, P.O. Box 4163, Berkeley, CA 94704-0163, U.S.A. The Pacific Journal of Mathematics is indexed by Mathematical Reviews, Zentralblatt MATH, PASCAL CNRS Index, Referativnyi Zhurnal, Current Mathematical Publications and Web of Knowledge (Science Citation Index).

The Pacific Journal of Mathematics (ISSN 0030-8730) at the University of California, c/o Department of Mathematics, 798 Evans Hall \#3840, Berkeley, CA 94720-3840, is published twelve times a year. Periodical rate postage paid at Berkeley, CA 94704, and additional mailing offices. POSTMASTER: send address changes to Pacific Journal of Mathematics, P.O. Box 4163, Berkeley, CA 94704-0163.

PJM peer review and production are managed by EditFLOW ${ }^{\circledR}$ from Mathematical Sciences Publishers.

\section{PUBLISHED BY}

\section{mathematical sciences publishers \\ nonprofit scientific publishing}

http://msp.org/

(C) 2015 Mathematical Sciences Publishers 


\section{PACIFIC JOURNAL OF MATHEMATICS}

Volume $276 \quad$ No. $1 \quad$ July 2015

On the degree of certain local $L$-functions

U. K. ANANDAVARdhanan and AmiYa Kumar MondaL

Torus actions and tensor products of intersection cohomology

ASILATA BAPAT

Cyclicity in Dirichlet-type spaces and extremal polynomials II: functions on the bidisk

Catherine Bénéteau, Alberto A. Condori, Constanze Liaw,

DANIEl SECo and Alan A. SOlA

Compactness results for sequences of approximate biharmonic maps

CHRISTINE BREINER and TOBIAS LAMM

Criteria for vanishing of Tor over complete intersections

Olgur Celikbas, Srikanth B. Iyengar, Greg PiePMeyer and

ROGER WIEGAND

Convex solutions to the power-of-mean curvature flow

\section{SHIBING CHEN}

Constructions of periodic minimal surfaces and minimal annuli in $\mathrm{Sol}_{3}$

CHRISTOPHE DESMONTS

Quasi-exceptional domains

ALEXANDRE EREMENKO and ERIK LUNDBERG

Endoscopic transfer for unitary groups and holomorphy of Asai $L$-functions

NEVEN GRBAC and FREYDOON SHAHIDI

Quasiconformal harmonic mappings between Dini-smooth Jordan domains

DAVID KALAJ

Semisimple super Tannakian categories with a small tensor generator

THOMAS KRÄMER and RAINER WEISSAUER

On maximal Lindenstrauss spaces

PETR PETRÁČEK and JiŘí SPURNÝ 